\title{
Status of the laboratory infrastructure for detector calibration and characterization at the European XFEL
}

\author{
N. Raab, ${ }^{a, 1}$ K.-E. Ballak, ${ }^{a}$ T. Dietze, ${ }^{a}$ M. Ekmedzič ${ }^{a}$ S. Hauf, ${ }^{a}$ F. Januschek, ${ }^{a}$ A. Kaukher, ${ }^{a}$ M. \\ Kuster, ${ }^{a}$ P. M. Lang, ${ }^{a}$ A. Münnich, ${ }^{a}$ R. Schmitt, ${ }^{a}$ J. Sztuk-Dambietz ${ }^{a}$ and M. Turcato ${ }^{a}$ \\ ${ }^{a}$ European XFEL GmbH, \\ Holzkoppel 4, Schenefeld, Germany \\ E-mail: natascha.raab@xfel.eu
}

\begin{abstract}
Aвstract: The European X-ray Free Electron Laser (XFEL.EU) will provide unprecedented peak brilliance and ultra-short and spatially coherent X-ray pulses in an energy range of 0.25 to $25 \mathrm{keV}$. The pulse timing structure is unique with a burst of 2700 pulses of 100 fs length at a temporal distance of $220 \mathrm{~ns}$ followed by a $99.4 \mathrm{~ms}$ gap. To make optimal use of this timing structure and energy range a great variety of detectors are being developed for use at XFEL.EU, including 2D X-ray imaging cameras that are able to detect images at a rate of $4.5 \mathrm{MHz}$, provide dynamic ranges up to $10^{5}$ photons per pulse per pixel under different operating conditions and covering a large range of angular resolution [3,4]. In order to characterize, commission and calibrate this variety of detectors and for testing of detector prototypes the XFEL.EU detector group is building up an $\mathrm{X}$-ray test laboratory that allows testing of detectors with X-ray photons under conditions that are as similar to the future beam line conditions at the XFEL.EU as is possible with laboratory sources [6]. A total of four test environments provide the infrastructure for detector tests and calibration: two portable setups that utilize low power X-ray sources and radioactive isotopes, a test environment where a commercial high power X-ray generator is in use, and a pulsed X-ray/electron source which will provide pulses as short as $25 \mathrm{~ns}$ in XFEL.EU burst mode combined with target anodes of different materials. The status of the test environments, three of which are already in use while one is in commissioning phase, will be presented as well as first results from performance tests and characterization of the sources.
\end{abstract}

KeYwords: X-ray detectors, detector alignment and calibration methods

\footnotetext{
${ }^{1}$ Corresponding author.
} 


\section{Contents}

1 Introduction 1

2 Portable vacuum compatible test setup PHEOBE 2

3 Portable ambient test setup Little Amber 3

4 Ambient setup for 1 mega pixel detectors Big Amber 4

5 Vacuum setup with pulsed electron source PulXar 6

6 Summary 6

$\begin{array}{lll}7 & \text { Acknowledgments } & 7\end{array}$

\section{Introduction}

The European X-ray Free Electron Laser is a high-brilliance fifth generation X-ray light source located in the area of Hamburg. It will provide spatially coherent X-rays in the energy range between 0 and $25 \mathrm{keV}$, delivered in 10 bursts of X-ray pulses per second, where each burst consists of up to 2700 pulses of $100 \mathrm{fs}$ length at a rate of $4.5 \mathrm{MHz}$ [1, 2]. Three $4.5 \mathrm{MHz}$ high rate 2D X-ray imaging cameras (Adaptive Gain Integrating Pixel Detector (AGIPD) [7], Large Pixel Detector (LPD) [8, 9] and DEPFET Sensor with Signal Compression (DSSC) [10-12] ), see figure 1) are being developed with the goal of acquiring single X-ray pulse images of 1 mega pixel size at the XFEL.EU. In addition small area 2D imaging cameras like the FastCCD [13] and pnCCD [14] will be used for imaging applications at the $10 \mathrm{~Hz}$ burst rate.
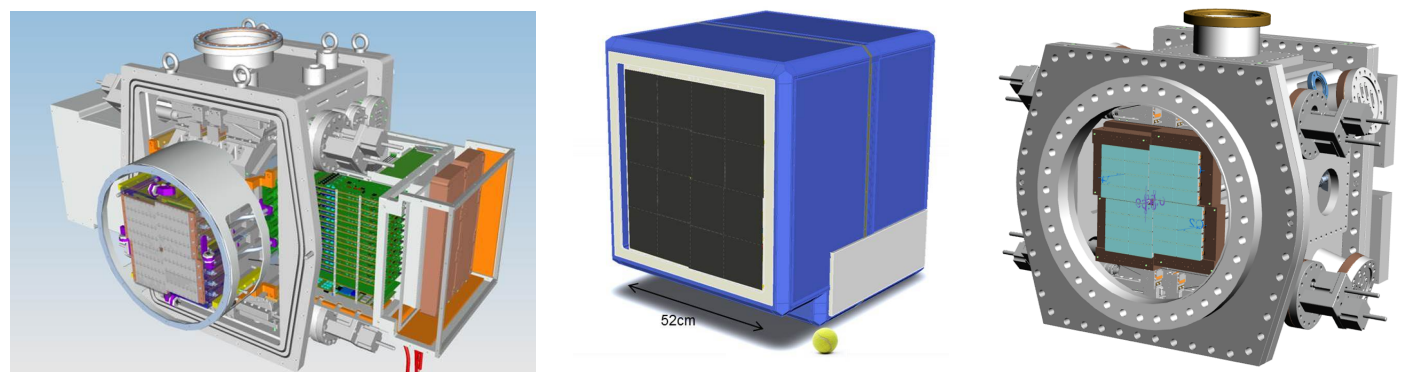

Figure 1: From left to right 3D CAD models of the AGIPD (image is courtesy by the AGIPD consortium), LPD (image is courtesy by the LPD consortium) and DSSC (image is courtesy by the DSSC consortium) 2D imaging detectors are shown including their mechanical housing (LPD) or vacuum setup (AGIPD, DSSC). 
In order to avoid beam time being used for characterization, commissioning and calibration a test laboratory has been established. The laboratory aims at testing detectors in conditions which are as close as possible to those in their final installations. The test setups developed must handle the large variety of detector differences (energy range, vacuum or air operation, pixel size, sensor area, acquisition rate, etc.) anticipated as defined by the following requirements.

Test setups are necessary that allow measurements of the detectors under ambient conditions as well as in vacuum at a pressure of $10^{-5}$ mbar. X-rays have to be provided at different energies between 0 and $25 \mathrm{keV}$ and with different illumination properties: point like illumination of single pixels is necessary as well as flat field illumination of large sensor areas to test for crosstalk and to apply flat field corrections. To test properties of the high rate cameras that depend on timing like the optimization of integration time or pulse to pulse afterglow, an X-ray source is required that can provide pulses in a time structure similar to that of the XFEL. To meet these requirements, four complementary test setups are being developed.

Two small size portable setups have been developed. PHEOBE operates under high vacuum and attaches to the vacuum vessel of the detector. Little Amber is built for tests of small detectors, like the LPD two-tile system, under ambient conditions. The setups are used for low intensity flat field and energy response measurements, whereby PHEOBE provides X-rays at lower and medium energies and Little Amber provides photon energies in the medium and higher range.

The Big Amber setup is large and allows test of the 1 mega pixel 2D imaging cameras. This setup is used for higher intensity flat field illumination of large sensor areas and beam spot illumination down to $50 \mu \mathrm{m}$ at higher photon energies.

Finally, the PulXar setup whose X-ray source provides pulses of $50 \mathrm{~ns}$ or shorter delivered at a rate of $4.5 \mathrm{MHz}$ in a burst of 2700 pulses in $600 \mu \mathrm{s}$, is needed for measurement of all high rate detectors and the setup has to be vacuum compatible and provide a wide range of photon energies.

Table 1 summarized the main parameters of the four setups, which are described in more detail in the following chapters.

Table 1: Parameters of the four different setups. As energies the positions of the main characteristic lines that are observed in the spectra are given

\begin{tabular}{|l|l|l|l|l|l|}
\hline Setup & $\begin{array}{l}\text { Operating } \\
\text { conditions }\end{array}$ & Energies $(\mathrm{keV})$ & $\begin{array}{l}\text { Intensity } \\
\left(\text { counts s-1 } \mathrm{sr}^{-1}\right)\end{array}$ & $\begin{array}{l}\text { Pulse } \\
\text { length }\end{array}$ & $\begin{array}{l}\text { Spot } \\
\text { size }\end{array}$ \\
\hline \hline Little Amber & Ambient & $5.9,9.7,20.2$ & $10^{8}$ for $20.2 \mathrm{keV}$ & n.a. & n.a. \\
\hline Big Amber & Ambient & $8,17.5$ & $10^{11}$ for $17.5 \mathrm{keV}$ & $168 \mathrm{~ns}$ & $50 \mu \mathrm{m}$ \\
\hline PHEOBE & $>10^{-6} \mathrm{mbar}$ & $5.9,9.7$ & $10^{7}$ for $9.7 \mathrm{keV}$ & n.a. & n.a. \\
\hline PulXar & $>10^{-8} \mathrm{mbar}$ & $0.3-17.5$ & to be measured & $50 \mathrm{~ns}$ & n.a. \\
\hline
\end{tabular}

\section{Portable vacuum compatible test setup PHEOBE}

The vacuum compatible setup PHEOBE is operated at pressures down to $5 \times 10^{-6}$ mbar and is attached to the vacuum vessel of a detector via a gate valve. A Programmable Logic Controller is 

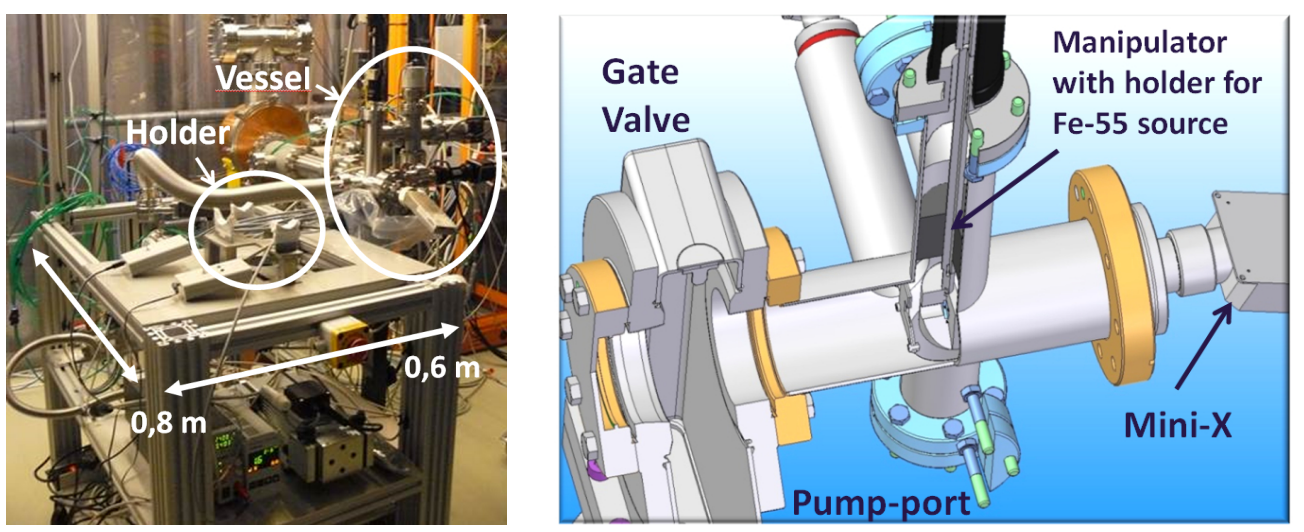

Figure 2: Left: PHEOBE setup is shown, consisting of its pump system and vacuum vessel attached to the pnCCD detector vessel in the background. Right: CAD rendering of the vessel including the manipulator with source holder and Mini-X is shown. Copyright The Author(s), published under the CC-BY 4.0 license (http://creativecommons.org/licenses/by/4.0/)

used to control the vacuum pumps, pressure gauges, valves and manipulators of the setup. Figure 2 shows the vacuum vessel of the setup, in which an Fe-55 source can be installed and moved by a linear translation stage in and out of the field of view of the detector without breaking the vacuum. At the end of a perpendicular manipulator a holder with filters attenuating the beam can be attached. Additionally a low power X-ray generator, an Amptek Mini-X [17] with Gold target, can be attached via a vacuum flange. The vacuum vessel provides shielding from X-rays up to $15 \mathrm{keV}$.

The emission spectra of the Fe-55 source, with an activity of $1.85 \mathrm{GBq}$, and the Mini-X are shown in figure 4. As with all spectra in this publication, it was obtained with a FastSDD detector from Amptek [15] (for the Fe-55 spectrum a highly transmissive low-energy window [16] was used.) PHEOBE is a very flexible setup for providing X-rays at lower and medium energies under vacuum conditions.

\section{Portable ambient test setup Little Amber}

The Little Amber setup is shown in figure 3, its shielding cabinet is $60 \mathrm{~cm} \times 120 \mathrm{~cm} \times 60 \mathrm{~cm}$ large, such that it can house detectors with sizes of up to approximately $0.5 \mathrm{~m} \times 0.5 \mathrm{~m} \times 0.5 \mathrm{~m}$. Inside the cabinet detectors are mounted on a breadboard in front of the source and forced ventilation is possible for prototypes with strong heat dissipation. The lead shielding of $1.5 \mathrm{~mm}$ thickness allows operation of the Amptek Mini-X tubes with accelerating voltages up to $50 \mathrm{kV}$. In addition to the X-ray tube with Gold target emitting characteristic X-rays of the $\mathrm{L}_{\alpha}$ and $\mathrm{L}_{\beta}$ transitions at $9.7 \mathrm{keV}$ and $11.4 \mathrm{keV}$, a Mini-X with Rhodium target (see figure 4) can be used which emits characteristic $\mathrm{K}_{\alpha}$ and $\mathrm{K}_{\beta}$ radiation at $20.2 \mathrm{keV}$ and $22.7 \mathrm{keV}$. For the measurement a FastSDD detector with a low-energy but light tight window was mounted at a distance of $18 \mathrm{~cm}$ in front of the Mini-X as can be seen in figure 3 .

Little Amber is extensively used for basic tests of small detectors or small prototypes of the $2 \mathrm{D}$ cameras. 

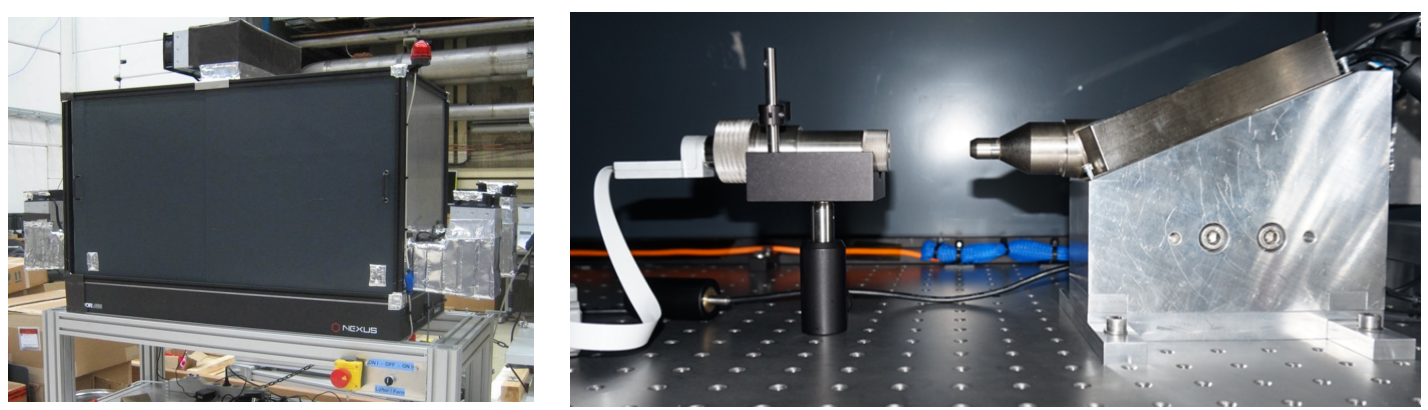

Figure 3: Left: the shielding cabinet of Little Amber with chicanes for ventilation. Right: a view inside showing the FastSDD detector mounted in front of the low power Xray generator (Mini-X). Copyright The Author(s), published under the CC-BY 4.0 license (http://creativecommons.org/licenses/by/4.0/)
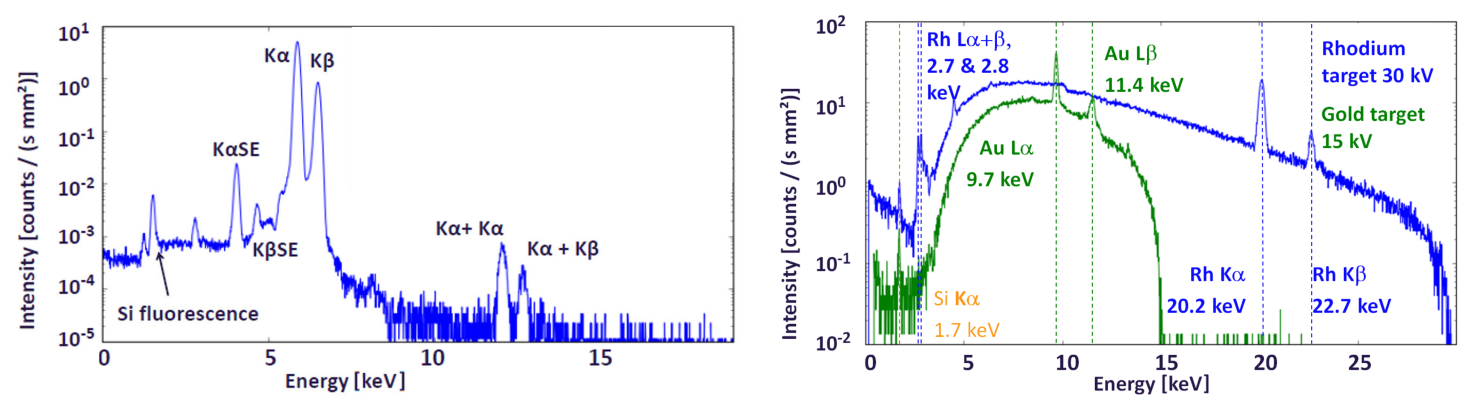

Figure 4: Left: X-ray emission spectrum of the Fe-55 source. As indicated the characteristic Mn $\mathrm{K}_{\alpha}$ and $K_{\beta}$ lines at 5.9 and $6.5 \mathrm{keV}$ can be observed, as well as the pile up peaks at the energies $E\left(K_{\alpha}\right)+E\left(K_{\alpha}\right)$ and $\left.E\left(K_{\alpha}\right)+E\left(K_{\beta}\right)\right)$, the $\mathrm{Si}$ escape peaks at $\left(\mathrm{K}_{\alpha} \mathrm{SE}\right.$ and $\left.\mathrm{K}_{\beta} \mathrm{SE}\right)$ and the fluorescence peak of the silicon detector material. Right: X-ray emission spectrum of the two Mini-X, that can be used in the Little Amber setup. For the Gold target characteristic X-ray L emission lines are visible, for the Rhodium target $\mathrm{K}$ - as well as the L-emission lines of the anode material can be observed. Both spectra show the $\mathrm{K}_{\alpha}$ peak of the silicon material of the detector. Copyright The Author(s), published under the CC-BY 4.0 license (http://creativecommons.org/licenses/by/4.0/)

\section{Ambient setup for 1 mega pixel detectors Big Amber}

The ambient test setup Big Amber is shown in figure 5, its shielding cabinet is $1.5 \mathrm{~m} \times 2 \mathrm{~m} \times 3.5$ $\mathrm{m}$ large, such that it can house the 1 mega pixel detectors that are presently foreseen to be used at the XFEL.EU. The shielding cabinet is divided into three sections. The first two consist of movable breadboard tables on which the frame and the shielding of $3 \mathrm{~mm}$ thick lead is mounted. Sliding doors give access to the inside. Both sections can be moved away from the third, the shielding of which reaches to the ground. One double door on the backside of the third section opens into a clean room and allows detectors to be easily moved into the setup once they are mounted on the detector support. Figure 5 also shows a CAD rendering of the third cabinet containing the vacuum vessel of the AGIPD 1 mega pixel detector mounted on the detector support.

Of all setups Big Amber can provide the highest intensity due to the Seifert high power X-ray 

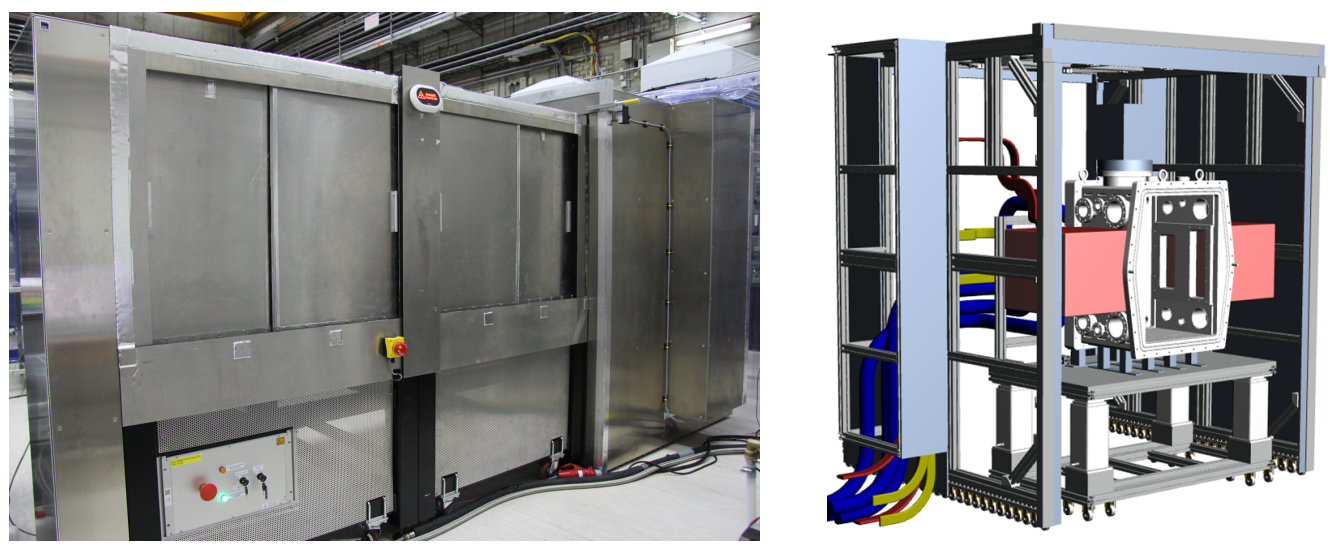

Figure 5: Left: shielding cabinet of the Big Amber. Right: a 3D CAD model rendering of the third cabinet containing the AGIPD vessel. Copyright The Author(s), published under the CC-BY 4.0 license (http://creativecommons.org/licenses/by/4.0/)

generator used. Tests requiring pulsed X-rays can be achieved using a high speed chopper, which produces pulses as short as $168 \mathrm{~ns}$.

The Seifert source is operated with Molybdenum or Copper anodes, at a voltage of up to 60 $\mathrm{kV}$ and power up to $2 \mathrm{~kW}$. X-ray tubes with different anodes are quickly exchangeable and give the opportunity to perform measurements at higher X-ray intensities with different photon energies. Filters to reduce the $\mathrm{K}_{\beta}$ radiation are included in the tube housing. Figure 6 shows the $\mathrm{X}$-ray emission spectra of a Molybdenum tube with characteristic $\mathrm{K}$ lines at $17.5 \mathrm{keV}$ and $19.6 \mathrm{keV}$, as well as the effect of Zirconium as K-edge filter on the shape of the emission spectrum. In this material the binding energy of the electron in the $\mathrm{K}$ shell of the atom lies with $\approx 18 \mathrm{keV}$ between the two peaks. Due to the rapid drop of the transmission coefficient at this energy [18], the intensity of the $\mathrm{K}_{\beta}$ radiation can be reduced effectively together with the underlying Bremsstrahlung continuum.
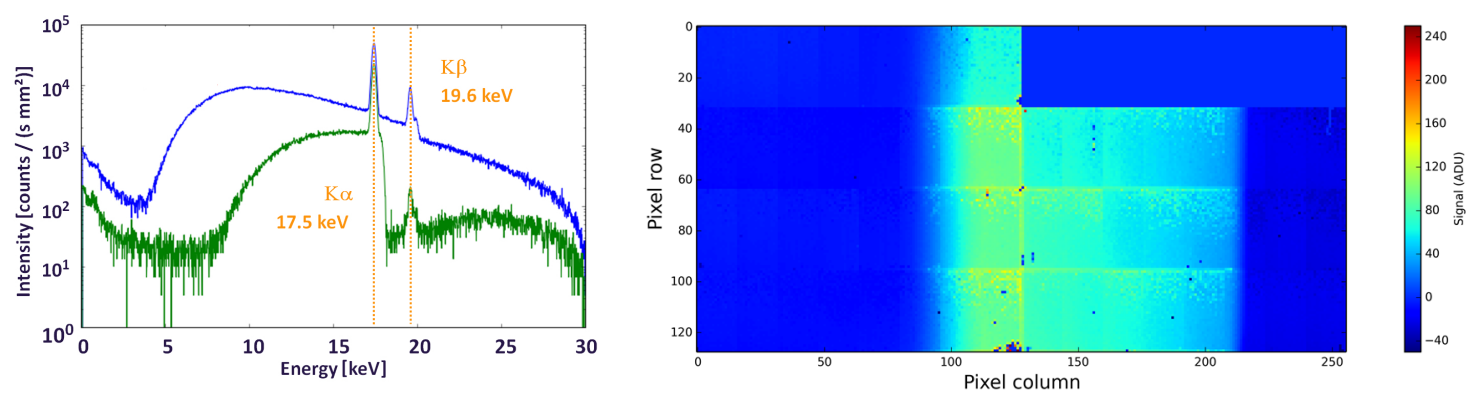

Figure 6: Left: X-ray emission spectrum of the Molybdenum tube without (blue) and with (green) a Zirconium filter. Right: Response of an LPD super module sensor illuminated with the Molybdenum tube at $50 \mathrm{kV}$ and $40 \mathrm{~mA}$ at a distance of $0.5 \mathrm{~m}$. The module was equipped with seven of eight possible tiles. Dead pixels are visible as well as the edges of the tiles where the pixels are 20\% larger and therefore detect a higher signal. Copyright The Author(s), published under the CC-BY 4.0 license (http://creativecommons.org/licenses/by/4.0/) 
Count rates of $10^{6}$ counts $\mathrm{mm}^{-2} \mathrm{~s}^{-1}$ can be provided with the unfocused beam at a distance of $0.5 \mathrm{~m}$. With an opening angle of approximately 8 degrees the beam spot at this distance allows illumination of several thousand pixels simultaneously, as shown for a small prototype of the LPD in figure 6, where offset corrected data from the sensor, irradiated with th Molybdenum X-ray tube, can be seen.

A focusing polycapillary optic from IFG can be installed to increase the photon intensity delivered to a pixel. This provides a focal spot of less than $50 \mu \mathrm{m}$ resulting in an intensity gain of up to $10^{4}$, depending on the photon energy, when compared to an unfocused beam. The commissioning of the optics is currently ongoing.

The Big Amber setup allows testing 1 Mpx Detectors with large-area illumination for flat field correction as well as scans of single pixels at medium or high photon energies.

\section{Vacuum setup with pulsed electron source PulXar}

The PulXar setup is currently being developed and will contain a pulsed electron source that is capable of producing pulses of 50-150 ns duration, within a $0.6 \mathrm{~ms}$ burst followed by a $99.4 \mathrm{~ms}$ gap [6]. A multi-target anode will allow generation of characteristic X-rays at energies between $0.3 \mathrm{keV}$ and $17.5 \mathrm{keV}$. In addition a filter wheel and polycapillary optics are foreseen.

The electron gun was obtained from Kimball with a customized blanker section to provide the necessary timing structure. With magnetic lenses the electron beam can be focused on the target material to be used. In figure 7 the electron beam can be seen on a phosphor screen attached to the beam outlet of the electron gun in case of an unfocused and a focused beam.

While the design of the setup components is ongoing, as a testbed a copy of the continuous multi target X-ray source, on loan from the Max-Planck Institute for extraterrestrial Physics (MPE), was assembled, commissioned and characterized. As an intermediate step towards the final PulXar setup the pulsed electron gun was combined with a target and filter wheel assembly provided by MPE, as seen in figure 7. Commissioning of this setup is currently in progress. PulXar will provide the unique opportunity for timing tests with a pulse structure similar to the European XFEL. Due to the use of a target wheel with multiple different target materials it will be possible to provide a pulsed beam for any photon energy that is needed for tests with the XFEL detectors.

\section{Summary}

While dedicated beam time at XFEL.EU for characterization, commissioning and calibration of detectors will still be mandatory, e.g. to test the full dynamic range of the $4.5 \mathrm{MHz} 2 \mathrm{D}$ cameras, the laboratory setups described will provide the possibility to carry out a variety of tests independent of beam time. The two portable setups PHEOBE and Little Amber are available for basic tests with detectors of smaller sensor size like the FastCCD and pnCCD. Big Amber has been used for tests with a smaller versions of AGIPD and LPD and is ready for measurements with the 1 mega pixel versions of these two detectors. Synchronization and timing tests will be realized with the customized pulsed electron source of the PulXar setup, that can provide a similar timing structure as the XFEL burst mode. While the final version of the PulXar setup is still in the design phase, a preliminary setup making use of the pulsed electron gun is under commissioning. 

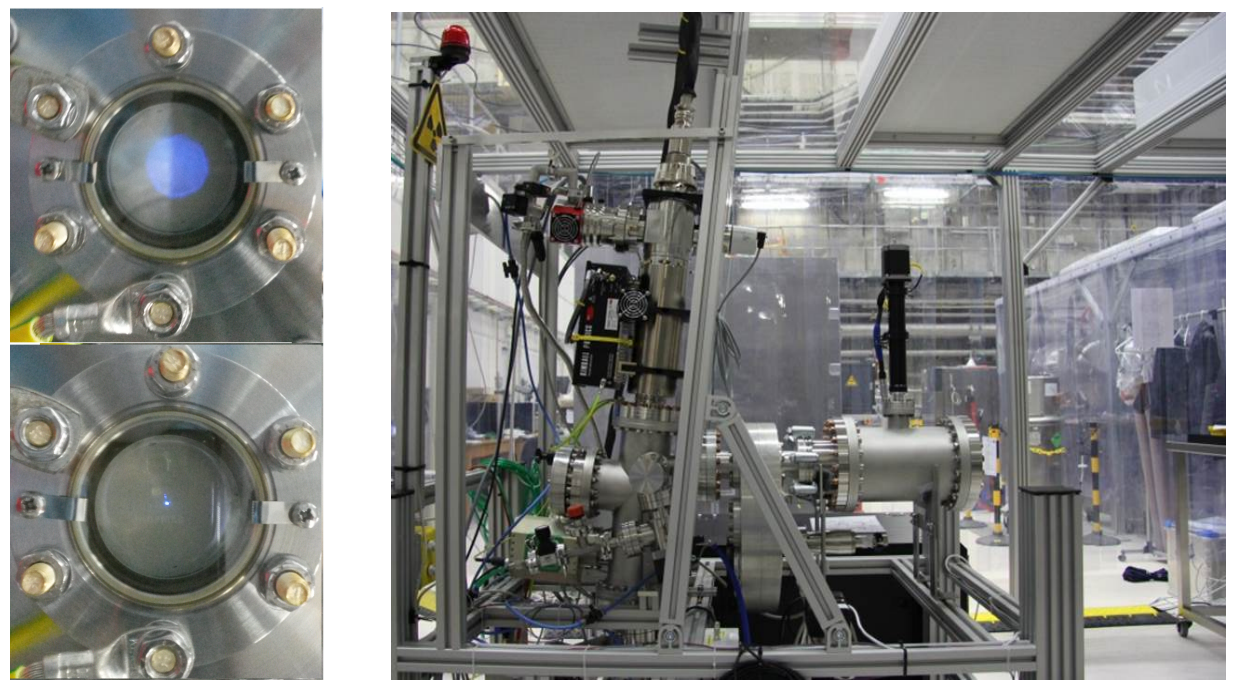

Figure 7: Left: beam spot of the unfocused beam (upper) and spot of focused beam (lower) with a lens current of $300 \mathrm{mV}$. Right: Vessel of the pulsed X-ray source PulXar with filter wheel provided by the MPE combined with the pulsed electron gun from Kimball. Copyright The Author(s), published under the CC-BY 4.0 license (http://creativecommons.org/licenses/by/4.0/)

\section{Acknowledgments}

The authors would like to thank the Max-Planck Institute for extraterrestrial Physics for providing an X-ray source with filter wheel for testing in our laboratory.

\section{References}

[1] Altarelli, M. et. al. , The European X-ray Free Electron Laser, European XFEL GmbH, Technical Design Report (2006), http://xfel.desy.de/localfsExplorer_read?currentPath=/afs/ desy.de/group/xfel/wof/EPT/TDR/XFEL-TDR-final.pdf

[2] Tschentscher, T. et al., Photon beam properties at the European XFEL, European XFEL GmbH, Technical Report TR-2011-006 (2012), doi:10.3204/DESY11-152

[3] Graafsma, H., Requirements for and development of 2 dimensional X-ray detectors for the European $X$-ray Free Electron Laser in Hamburg, JINST (2009)

[4] Kuster, M. et. al., Detectors and Calibration Concept for the European XFEL, Synchrotron radiation news 27 (2014) 35 - 38

[5] Sztuk-Dambietz, J., et al., Status of detector development for the European XFEL, SPIE Optics+ Optoelectronics, International Society for Optics and Photonics (2013)

[6] Sztuk-Dambietz, J., et al. Laboratory Infrastructure for Detector Calibration Characterization at the European XFEL, Nuclear Science Symposium and Medical Imaging Conference (NSS/MIC), IEEE (2013).

[7] B. Henrich, et.al., The adaptive gain integrating pixel detector AGIPD a detector for the European XFEL, Nucl. Instr. and Meth. A (2010), doi:10.1016/j.nima.2010.06.107 
[8] Hart, M. et al., Development of the LPD, a high dynamic range pixel detector for the European XFEL, Proceedings of the Nuclear Science Symposium and Medical Imaging Conference (NSS/MIC), IEEE, (2012)

[9] Koch, A. et al., Performance of an LPD prototype detector at MHz frame rates under Synchrotron and FEL radiation Journal of Instrumentation, 8 (2013)

[10] Porro, M. et al., Development of a DEPFET sensor with signal compression: A large format X-ray imager with mega-frame readout capability for the European XFEL, IEEE Trans. Nucl. Sci. Vol. 59, No 6 (2012) 3339.

[11] Porro, M. et al., Expected performance of the DEPFET sensor with signal compression: A large format X-ray imager with mega-frame readout capability for the European XFEL, Nucl. Instrum. Methods, A624 (2010) 509.

[12] G. Lutz et al., DEPFET sensor with intrinsic signal compression developed for the use at the XFEL free electron laser radiation source, Nucl. Instrum. Methods A624, (2010) 528

[13] P. Denes, et al. A fast, direct X-ray detection charge-coupled device, Rev. Sci. Instrum., 80(083302), 2009.

[14] Hartmann, R. et. al., Large format pnCCDs as imaging detectors for X-ray free-electron-lasers, IEEE Nuclear Science Symposium Conference Record, (2008) 2590-2595

[15] Fast SDD®Specifications, AMPTEK INC., http: //amptek. com/wp-content/uploads/2016/07/Fast-SDD-Specifications.pdf

[16] Amptek C series windows, AMPTEK INC., www . amptek.com/pdf/lew_faq.pdf

[17] Miniature X-ray Tube for OEM Applications, AMPTEK INC., http: //www . amptek . com/pdf/minixoem.pdf

[18] X-ray Data Booklet (October 2009) http: //xdb. lbl.gov/xdb-new.pdf 